\title{
Non-cardiac benefits of human recombinant erythropoietin in end stage renal failure and anaemia
}

\author{
K P Morris, J Sharp, S Watson, M G Coulthard
}

\begin{abstract}
Recombinant human erythropoietin (r-HuEpo) is now available to correct the anaemia of end stage renal failure. The clinical consequences of increasing the haemoglobin concentration in children on dialysis are incompletely documented; a placebo controlled study is essential when assessing subjective changes, for example in appetite or other aspects of quality of life.
\end{abstract}

A single blind, placebo controlled crossover study in 11 children with end stage renal failure was performed to assess the clinical benefits resulting from correction of anaemia. Ten of the 11 children completed 36 weeks of the study and seven completed both 24 week limbs. Subcutaneous administration of $\mathbf{r}-\mathrm{HuEpo}$ twice a week resulted in an increase in haemoglobin concentration, from 73 to $112 \mathrm{~g} / \mathrm{l}$. This was associated with an objective improvement in exercise tolerance, and a subjective improvement in physical performance and health, and better school attendance. No consistent effect was seen on appetite, growth, psychosocial functioning, biochemical control, or peritoneal dialysis efficiency. A small but clinically unimportant increase in systolic and diastolic blood pressure was seen in five children. One child on antihypertensive treatment required an increase in dosage during r-HuEpo while another child required a reduction in treatment.

These findings, together with the important cardiac benefits previously described during r-HuEpo treatment, support the use of $\mathbf{r}-\mathrm{HuEpo}$ in all children with end stage renal failure and anaemia. (Arch Dis Child 1993; 69: 580-586)

A deficiency of erythropoietin is the single most important factor contributing to anaemia in end stage renal failure. Anaemia may be responsible for several of the clinical features of end stage renal failure in children, such as reduced exercise tolerance, fatigue, poor appetite, and impaired growth. With the characterisation and manufacture of recombinant human erythropoietin ( $r$-HuEpo) in recent years it has become possible to correct anaemia and to assess which of these clinical features are attributable to anaemia.

An extensive list of benefits of $r-H u E p o$ have been reported in adult patients. ${ }^{1}$ Although a limited number of studies of r-HuEpo in children with end stage renal failure have confirmed its efficacy in correcting anaemia, ${ }^{2-4}$ none of these has been placebo controlled, making it impossible to interpret critically many of the effects ascribed to r-HuEpo. The present study was therefore designed as a single blind, placebo controlled crossover study in order to remove any bias that undoubtedly exists towards a new drug as heavily publicised as r-HuEpo and to assess the effects of r-HuEpo on quality of life, diet, exercise tolerance, and peritoneal dialysis efficiency in a group of children with end stage renal failure on long term peritoneal dialysis. The cardiac benefits of r-HuEpo have been previously reported. ${ }^{5}$

\section{Patients and methods}

Eleven children (nine boys, two girls) were enrolled into the study. Their median age was 6.7 years (range $2 \cdot 3-12 \cdot 3$ ), and median haemoglobin concentration $73 \mathrm{~g} / 1$ (range 42-81). The range of underlying conditions and other clinical details are summarised in the table. Ten of the children were on dialysis at the time of the study: peritoneal rapid overnight dialysis in nine cases and haemodialysis in one. Five children were receiving treatment for hypertension but all were normotensive, assessed with regular home blood pressure monitoring (Waeschle DS40).

The design was a single blind, placebo controlled, crossover study with two equal limbs of 24 weeks' duration. The study was approved by the Newcastle health authority ethics committee. Six children were allocated by stratified randomisation to receive $\mathrm{r}-\mathrm{HuEpo}$ for 24 weeks followed by placebo (group 1; table), and five the reverse treatment order (group 2; table). r-HuEpo (Recormon, Boehringer Mannheim) or placebo were administered subcutaneously twice a week by the child or parent at home. The volume of all injections was fixed at $0.5 \mathrm{ml}$. r-HuEpo was commenced at $50 \mathrm{U} / \mathrm{kg} /$ week and increased in stepwise increments to a maximum of 400 $\mathrm{U} / \mathrm{kg} /$ week by week 12 , and thereafter adjusted to maintain a target haemoglobin concentration of $105-120 \mathrm{~g} / \mathrm{l}$. In some cases this was achieved with a once weekly subcutaneous injection of r-HuEpo; this was balanced by a similar regimen during the placebo limb to avoid a treatment difference between limbs. Seven children completed both 24 week limbs of the study, two completed the r-HuEpo limb and half of the placebo limb, one completed the placebo limb and half of the r-HuEpo limb, 
Clinical details of the 11 children entering the study

\begin{tabular}{|c|c|c|c|c|c|c|c|c|}
\hline $\begin{array}{l}\text { Patient } \\
\text { No }\end{array}$ & Sex & $\begin{array}{l}\text { Age } \\
\text { (years) }\end{array}$ & $\begin{array}{l}\text { Haemoglobin } \\
(\mathrm{g} / \mathrm{l})\end{array}$ & $\begin{array}{l}\text { Dialysis } \\
\text { duration } \\
\text { (years) }\end{array}$ & $\begin{array}{l}\text { Mode } \\
\text { of } \\
\text { dialysis }\end{array}$ & Diagnosis & $\begin{array}{l}\text { Treatment } \\
\text { for } \\
\text { hypertension }\end{array}$ & $\begin{array}{l}\text { No of } \\
\text { weeks in } \\
\text { study }\end{array}$ \\
\hline \multicolumn{9}{|c|}{ Group 1 (r-HuEpo/placebo) } \\
\hline 1 & $\mathbf{M}$ & $10 \cdot 4$ & 42 & $3 \cdot 2$ & PROD & Familial HUS & Yes & 48 \\
\hline 2 & $\mathbf{M}$ & $2 \cdot 8$ & 72 & $2 \cdot 5$ & PROD & Dysplasia & No & 48 \\
\hline 3 & $\mathrm{~F}$ & $4 \cdot 5$ & 81 & $3 \cdot 8$ & PROD & Dysplasia & No & 48 \\
\hline 4 & $\mathrm{M}$ & $3 \cdot 0$ & 80 & 1.7 & PROD & $\begin{array}{l}\text { Congenital nephrotic } \\
\text { syndrome }\end{array}$ & Yes & 48 \\
\hline 5 & $\mathbf{M}$ & $6 \cdot 4$ & 73 & $3 \cdot 1$ & Haemodialysis & $\begin{array}{l}\text { Posterior urethral } \\
\text { valves }\end{array}$ & No & 40 \\
\hline 6 & F & $8 \cdot 9$ & 66 & $0 \cdot 4$ & PROD & Unknown & Yes & 36 \\
\hline \multicolumn{9}{|c|}{ Group 2 (placebo/r-HuEpo) } \\
\hline 7 & $\mathbf{M}$ & $12 \cdot 3$ & 76 & 0.4 & PROD & FSGS & Yes & 38 \\
\hline 8 & $\mathbf{M}$ & $8 \cdot 8$ & 68 & 0 & None & $\begin{array}{l}\text { Reflux associated } \\
\text { dysplasia }\end{array}$ & No & 48 \\
\hline 9 & $\mathbf{M}$ & $6 \cdot 7$ & 76 & $3 \cdot 2$ & PROD & Dysplasia & No & 48 \\
\hline 10 & $\mathbf{M}$ & $2 \cdot 3$ & 64 & $1 \cdot 0$ & PROD & Recessive PCKD & Yes & 30 \\
\hline 11 & $\mathbf{M}$ & $8 \cdot 8$ & 75 & $7 \cdot 9$ & PROD & $\begin{array}{l}\text { Posterior urethral } \\
\text { valves }\end{array}$ & No & 48 \\
\hline
\end{tabular}

$\mathrm{PROD}=$ peritoneal rapid overnight dialysis; $\mathrm{HUS}=$ haemolytic uraemic syndrome; $\mathrm{PCKD}=$ polycystic kidney disease; FSGS = focal segmental glomerulosclerosis.

* Patient with Duchenne muscular dystrophy.

and one completed only the placebo limb (table). Three children withdrew because they underwent renal transplantation and one child died of hepatic fibrosis, related to underlying polycystic disease.

\section{EXERCISE TOLERANCE}

This was assessed in two ways. Firstly, the 2 minute walking distance was measured. ${ }^{6}$ Each child was asked to walk as fast as possible up and down an enclosed section (25 metres) of hospital corridor with no gradient. Simple encouragement was given and, in view of a possible training effect, the greater of two distances was used in the analysis. Seven of the 11 children were judged old enough to participate.

Secondly, six of the older children also underwent formal treadmill exercise testing (Marquette instruments) using either a Bruce protocol $(n=3)$ or a modified Bruce protocol $(n=3) .{ }^{7}$ The duration of exercise and the total workload (calculated by Marquette software) was recorded in addition to the heart rate response to exercise and the electrocardiographic waveform was analysed for evidence of ischaemia or arrhythmia.

\section{QUALITY OF LIFE}

This was assessed by means of a 25 part parental questionnaire, applying a modified format of a previously used questionnaire (appendix). ${ }^{8}$ The questions covered various aspects of the child's well being and behaviour including mood and psychological behaviour, social interaction, somatic complaints and general health, sleep, diet, school functioning, and physical performance. Parents completed the questionnaire during the two week run-in period before the study and at the end of each limb. A visual analogue scale $(10 \mathrm{~cm})$ was used to score each question, with the best and worst possible responses at each end of the scale. Parents were allowed to see their previous assessments and movements of less than $5 \mathrm{~mm}$ away from the previous mark were ignored. Each of the first 23 questions was analysed separately and then those questions covering a common area, such as physical performance and health (Q6, 7, 15, 18, 22, 23), diet (Q10-12), psychosocial behaviour $(\mathrm{Q} 5,8,9$, $13,14,16,17,19)$, school performance (Q20, 21 ), and sleep behaviour (Q1-4) were grouped together. Finally, the global score, incorporating all 23 questions, was calculated. In addition a further question (Q24) was included to assess, in as general a way as possible, whether the parents had observed any overall improvement in the child during either treatment period. Finally, at the end of the study each parent was asked to identify the treatment order that their child had received.

\section{DIETARY INTAKE}

A prospective three day dietary diary of all food consumed was completed by the parents during the two week run-in period before the study and every 12 weeks during the study. Each parent was asked to record all food and drink taken in terms of type and quantity, including nasogastric feed in the four children on overnight nasogastric tube feeding. This type of assessment is very familiar to these parents, forming part of routine patient management. A senior dietitian (SW), who was unaware of the treatment order, calculated the mean daily energy, protein, potassium, and phosphate intake for each period.

\section{ANTHROPOMETRIC MEASUREMENTS}

Every four weeks weight, height, triceps subcutaneous fat thickness (Harpenden Skinfold Caliper, British Indicators, Luton) and midarm circumference were measured. Weight, height, and mid-arm circumference velocity were calculated for each limb by fitting a regression line through all data points and these velocities were used in the analysis.

PERTTONEAL DIALYSIS STUDY

Peritoneal dialysis efficiency was assessed using a 30 minute equilibration study at the end of each limb of the study. ${ }^{9}$ This involves running a known volume of dialysis fluid into an empty peritoneal cavity and taking two timed dialysis fluid samples over a period of 30 
minutes, together with a single blood sample. Measurement of potassium, urea, creatinine, glucose, and phosphate on each specimen allows estimation of the half life for equilibration across the peritoneal membrane for each substance. In addition the volume of ultrafiltrate removed after each overnight dialysis was recorded and the mean of 10 consecutive nights calculated at the end of each limb.

\section{LABORATORY MEASUREMENT}

Full blood count was measured every two weeks using a Coulter counter ('STKS', Coulter Electronics, Luton). Several measures of iron status were taken and the results have been reported in detail elsewhere. ${ }^{10}$ Plasma viscosity, creatinine and electrolytes, calcium, phosphate, bilirubin, aspartate transaminase, alkaline phosphatase, albumin, and parathyroid hormone were also measured regularly. Serial r-HuEpo concentrations were measured a constant interval after the last dose in each child; this interval ranging from 2.5 to 4 days in different children. Serum r-HuEpo concentration was measured using a radioimmunoassay (Boehringer Mannheim).

\section{BLOOD PRESSURE}

Each child had their resting blood pressure recorded twice a week at home using an automatic blood pressure machine (Waeschle DS40). The same site of measurement and cuff size was used throughout in each child.

\section{STATISTICAL ANALYSIS}

Treatment effects were calculated using statistical methods appropriate for the analysis of crossover trials, after excluding a significant period effect. ${ }^{11}$ To test for the presence of a treatment effect the difference in any measured variable between week 24 (end of period 1) and week 48 (end of period 2) is calculated, and the mean difference found in group $1 \mathrm{com}-$ pared with that found in group 2 , using a standard two sample $t$ test. In the absence of a treatment effect the differences are the same and the $t$ test non-significant.

Certain variables were only measured at the end of each 24 week limb (treadmill exercise test, peritoneal dialysis study) and the analysis therefore includes only those children completing both limbs of the study. Other
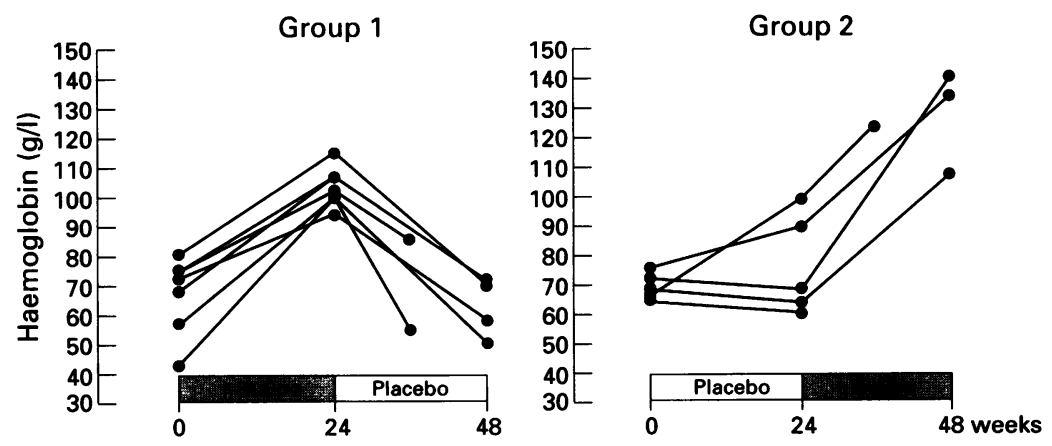

Figure 1 Individual changes in haemoglobin in all 11 children entered into the study. variables, however, were measured every 12 weeks throughout the study ( 2 minute walking distance, quality of life questionnaire, dietary intake) and it was therefore possible to include data from the three children who completed 36 weeks of the study but failed to complete both limbs. For these variables the mean of the measurements taken during each limb was used in the analysis. Blood pressure readings from these children were also included in the analysis. We felt that use of their data was justified because after 12 weeks of r-HuEpo their haemoglobin concentrations had already risen significantly (from 72 to $99 \mathrm{~g} / \mathrm{l}$ ) and within 12 weeks of stopping r-HuEpo their haemoglobin concentration had fallen to baseline values (from 103 to $67 \mathrm{~g} / \mathrm{l}$ ). The Wilcoxon signed rank sum test was used to assess the responses to the individual questions on the questionnaire while the group data is presented as the confidence interval for the difference between the treatment limbs, after summing the responses to related individual questions.

To analyse the weight, height, and mid-arm circumference data a within-patient comparison was made of the velocities during both limbs using a paired $t$ test. A within-patient comparison is vital in view of the large variability in background growth velocities in different children. Lastly, the possibility of a transient effect of r-HuEpo on certain laboratory variables (white cell count, platelet count, serum r-HuEpo concentration) was assessed by looking separately at sequential data from the nine children who completed a 24 week limb of r-HuEpo.

\section{Results}

\section{HAEMATOLOGICAL RESPONSE}

The median haemoglobin rose from $73 \mathrm{~g} / 1$ (range 42-81) at baseline to $112 \mathrm{~g} / \mathrm{l}$ (range

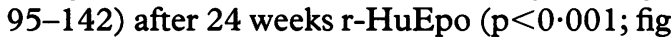
1). Five children developed evidence of iron deficiency but responded to iron supplementation; four responded to oral treatment but one child was intolerant of oral iron and required intravenous iron. ${ }^{10}$ Once the target haemoglobin had been reached it was possible to maintain it in four of the children on a once weekly dose regimen. At the end of the 24 week $\mathrm{r}$-HuEpo limb the mean weekly maintenance dose was $196 \mathrm{U} / \mathrm{kg}$. No significant change in platelet count, white cell count, or plasma viscosity was found during r-HuEpo.

\section{BIOCHEMISTRY}

No significant change occurred in sodium, potassium, urea, creatinine, calcium, phosphate, albumin, bilirubin, aspartate transaminase, alkaline phosphatase, or parathyroid hormone concentration.

\section{EXERCISE TESTING}

The 2 minute walking distance increased during the r-HuEpo limb in the seven children in whom data from both limbs is available $(p=0.06$; fig $2 A)$. Only three children 

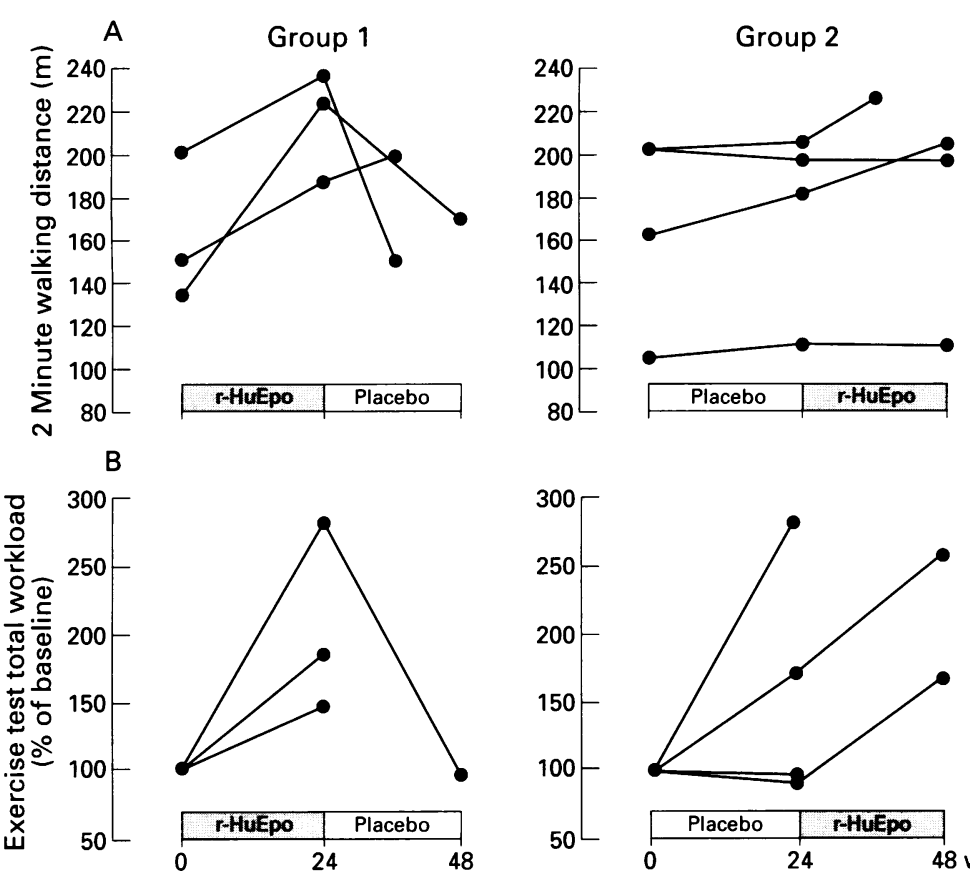

Figure 2 Changes in exercise tolerance measured by (A) 2 minute walking distance $(n=7)$ and $(B)$ total workload during treadmill exercise testing (expressed as a percentage of baseline $)(n=6)$. attributed to r-HuEpo, though total energy intake tended to increase $(p=0 \cdot 19)$. Repeat analysis after adjustment for nasogastric feed intake produced a similar result. Individual parents, however, noticed a marked improvement in appetite during $\mathrm{r}-\mathrm{HuEpo}$ which disappeared during placebo.

\section{ANTHROPOMETRIC MEASUREMENT}

No significant increase in weight $(p=0 \cdot 21)$ or height velocity $(p=0 \cdot 21)$ was seen during $\mathrm{r}$-HuEpo though again individual children showed an increased weight velocity during $\mathrm{r}$-HuEpo relative to placebo. An increase in mid-arm circumference velocity was seen during r-HuEpo, though this failed to reach statistical significance $(p=0 \cdot 09)$. There was no comparable increase in triceps skinfold thickness though there was a greater variability in results, largely attributable to poor cooperation in some of the younger children who found the procedure uncomfortable.

\section{PERITONEAL DIALYSIS STUDY}

No change in the peritoneal equilibration of potassium, urea, phosphate, glucose or creatinine, or in the ultrafiltrate volume after overnight dialysis, occurred during r-HuEpo.

completed treadmill exercise testing during both limbs; compared with placebo a marked increase in workload was seen during r-HuEpo $(p=0.07$; fig $2 B)$. A change in the heart rate response to exercise was seen after $r-H u E p o$ with a reduction in heart rate at rest $(p=0.002)$ and at each successive stage of the exercise test. No arrhythmias or ischaemic changes were seen during either limb.

\section{QUALITY OF LIFE}

Four of the 23 individual questions scored significantly better during $r-\mathrm{HuEpo}(\mathrm{p}<0.05$; numbers $6,15,18,23)$. In addition the response to the final question (number 24) suggested an overall improvement in the children during $\mathrm{r}-\mathrm{HuEpo}(\mathrm{p}<0.05)$.

After grouping related questions together an improvement was found in those questions relating to physical performance and general health, including a better school attendance $(p<0.02)$. No significant change was found in the other areas. Figure 3 shows the change in group scores after r-HuEpo, represented as confidence intervals for the difference between r-HuEpo and placebo. The global score, incorporating all questions, showed an increase though this did not reach significance $(p=0 \cdot 18)$.

Of the seven children who completed both limbs of the study five sets of parents identified the treatment order that their child had received, and all were correct. Two sets of parents were unable to detect a noticeable difference between limbs, despite increases in haemoglobin from 68 to $142 \mathrm{~g} / 1$ and from 64 to $109 \mathrm{~g} / 1$.

\section{DIETARY INTAKE}

No significant change in total energy, protein, potassium, or phosphate intake could be

\section{BLOOD PRESSURE}

Complete blood pressure profiles were available in only seven of the children because the other parents did not keep a consistent written record. Mean values were calculated from 10 consecutive readings at the end of each limb or, in three cases, from the last 10 readings before withdrawing from the study. Of the four children already on antihypertensive treatment one required an increase and one a reduction in therapy. Analysis of the remaining five children, three of whom were not on antihypertensive treatment during the study, showed an increase in systolic blood pressure during $r-H u E p o(p<0 \cdot 05)$. However, this was a small increase (maximum $6.4 \%$ ) and did not require any change in treatment. Mean diastolic blood pressure also increased slightly in the three children in whom it was recorded.

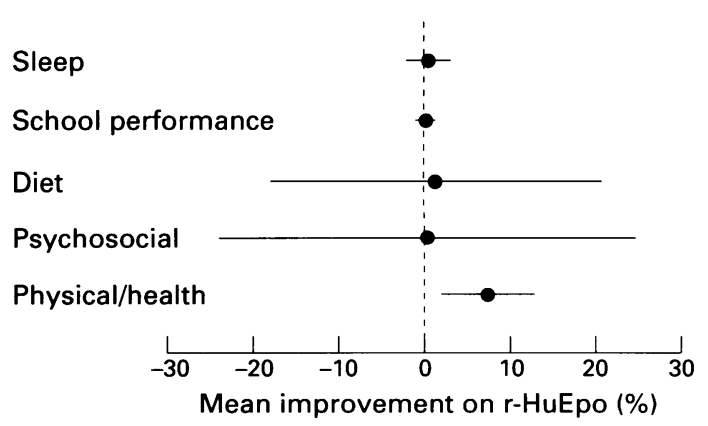

Figure 3 Quality of life questionnaire responses after grouping related questions $(n=10)$. Presented as the mean percentage difference after $r$-HuEpo relative to placebo, with the $95 \%$ confidence intervals for the difference. 


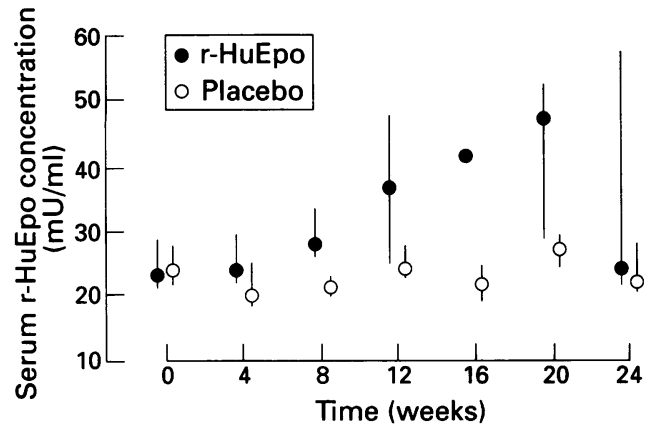

Figure 4 Sequential serum $r$-HuEpo concentrations during the placebo $(n=9)$ and $r-H u E p o(n=9)$ limbs. Median values and interquartile ranges shown.

SERUM r-HUEPO CONCENTRATION

Over the course of the 24 weeks of r-HuEpo treatment there was a progressive increase in serum r-HuEpo concentration, though there was considerable individual variation. By comparison serum r-HuEpo concentration remained fairly constant during the placebo limb (fig 4).

\section{Discussion}

Approximately 120 children in the UK are currently in end stage renal failure and on dialysis. The vast majority are anaemic and, until very recently, many were transfusion dependent, exposing them to the risks of viral transmission, iron overload, and antigen sensitisation. It is now clear that r-HuEpo is able to reverse the anaemia in virtually all cases, though various factors, in particular iron deficiency, may reduce its efficacy. r-HuEpo is able to abolish transfusion dependency with obvious advantages, including a reduction in HLA sensitisation ${ }^{12}$ and the potential of easier transplantation. What is much less clear, however, is what other benefits can be attributed to r-HuEpo. Too many studies have ignored the need for a placebo controlled design particularly when assessing subjective changes, for example in quality of life, amid the positive publicity that surrounded the introduction of $\mathrm{r}-\mathrm{HuEpo}$ into clinical practice.

Undertaking a placebo controlled study of $r-H u E p o$ in children is difficult because parents and children need to consent to a prolonged period of placebo injections. In addition each centre has only a small number of eligible patients. Although this was a small study, we feel that the inclusion of a placebo period and the crossover design provided more meaningful data than could have been obtained from a much larger uncontrolled study. Previous studies in children have al been uncontrolled and have predominantly involved older children given intravenous $\mathrm{r}$-HuEpo at the end of haemodialysis. We were able to confirm that subcutaneous administration of r-HuEpo twice a week is able to correct anaemia once an adequate supply of iron is present. ${ }^{10}$ No change in platelet count was found during this study, in contrast with previous studies, in both adults ${ }^{13}$ and children, ${ }^{14}$ which documented an early increase in platelet count on r-HuEpo. These studies involved intravenous administration of $\mathrm{r}$-HuEpo, which has a very different pharmacokinetic profile and achieves a much higher peak plasma concentration than subcutaneous r-HuEpo. ${ }^{15} 16$

Important cardiac benefits of short term $r$-HuEpo treatment in this cohort of patients have already been reported, ${ }^{5}$ with a reduction in cardiac output and ventricular hypertrophy. In addition correction of anaemia is able to improve aspects of quality of life and to have a beneficial effect on exercise tolerance.

The specific questions within the quality of life questionnaire showing significant improvements after $\mathrm{r}-\mathrm{HuEpo}$ were those reflecting less fatigue and breathlessness during exercise, improved general health, and better school attendance. In addition the final question, which attempted to summarise any overall change for the better on r-HuEpo, scored significantly better. Grouping related questions together confirmed an improvement in physical performance and health but failed to show any significant change in psychosocial, diet, school, or sleep behaviour. These findings are very similar to a large double blind study in adult dialysis patients looking at quality of life, which found an improvement in fatigue, strength, and physical symptoms without a consistent effect on psychological functioning or sleep disturbance. ${ }^{17}$ Although the majority of parents were able to correctly identify their child's r-HuEpo/placebo treatment order, two sets of parents were unable to do so despite a dramatic rise in haemoglobin, reflecting how well some children are able to compensate for severe anaemia. One of these children, however, had muscular dystrophy and clearly was not able to benefit from the improved physical performance seen in the other children.

Previous studies in children have documented improved exercise tolerance and an increase in peak oxygen consumption and ventilatory anaerobic threshold after r-HuEpo. ${ }^{3} 1218$ The importance of including a control group was, however, shown in one of these studies in which the control group also showed a significant improvement in exercise tolerance over the 16 week observation period. ${ }^{18}$ In our study both measures of exercise tolerance showed clear improvement after $\mathrm{r}-\mathrm{HuEpo}$, although the small number of children prevented this reaching statistical significance.

Previous uncontrolled studies in children have suggested an improvement in appetite associated with $\mathrm{r}-\mathrm{HuEpo},{ }^{19}$ but we were unable to confirm this with either the record of dietary intake, the parental assessment of appetite on the questionnaire, or with changes in weight velocity. Individual parents, however, commented on marked improvements in appetite during r-HuEpo. In addition an increase in mid-arm circumference velocity was seen. It is possible therefore that a large study might be able to document an improvement in appetite and weight velocity. $\mathrm{r}$-HuEpo did not result in any improvement in height velocity, confirming previous observations, ${ }^{20}$ and making it unlikely that anaemia contributes to the growth failure of end stage renal failure. 
An increase in packed cell volume, coupled with a reduction in cardiac output, could theoretically reduce peritoneal dialysis efficiency by reducing effective plasma flow to the peritoneum. In practice our study, and the majority of studies in adults, 2122 found no change in either dialysis efficiency or in ultrafiltrate volume, though others have reported an increase in ultrafiltrate, ${ }^{23}$ with either an increase $\mathrm{e}^{23}$ or decrease ${ }^{24}$ in solute transport. In keeping with an unchanged dietary intake and peritoneal dialysis efficiency we found no change in plasma potassium, urea, creatinine, or phosphate concentrations during r-HuEpo treatment.

The development, or exacerbation, of hypertension is the commonest reported side effect of $\mathrm{r}$-HuEpo administration, on occasions leading to encephalopathy. ${ }^{25}$ Patients with pre-existing hypertension at the start of treatment may be particularly at risk. During this study only one child with pre-existing hypertension required a marked increase in antihypertensive treatment during r-HuEpo. By contrast a minority of patients actually show a fall in blood pressure on r-HuEpo ${ }^{326}$; one child required a marked reduction in antihypertensive treatment during the r-HuEpo limb, probably as a result of a fall in cardiac output, followed by an increase in treatment during the placebo limb once his haemoglobin had fallen again. In the remaining children a small increase in both systolic and diastolic blood pressure was seen, though this did not necessitate any change in antihypertensive treatment. This increase in blood pressure was not evident in a previous analysis of this patient group, ${ }^{5}$ in which a single blood pressure reading only was taken at the time of echocardiography, stressing the importance of repeated measurement to minimise the inherent variability of blood pressure.

Although the bioavailability of $\mathrm{r}-\mathrm{HuEpo}$ after subcutaneous injection is only $21-40 \%$ that of intravenous r-HuEpo, ${ }^{15} 16$ its longer half life allows a more sustained action and produces a greater erythropoietic response for an equivalent dose. ${ }^{27}$ The absorption and elimination of subcutaneous r-HuEpo does not appear to change with long term treatment. ${ }^{28}$ The progressive increase in serum $\mathrm{r}-\mathrm{HuEpo}$ concentration seen over the course of the $\mathrm{r}$-HuEpo limb is compatible with published pharmacokinetic data in children. ${ }^{15} \mathrm{~W}$ ith a half life of 21 hours the serum concentration will remain significantly above the predose concentration for at least four days after a subcutaneous dose of $r-H u E p o$. If the bone marrow sensitivity to $\mathrm{r}-\mathrm{HuEpo}$ remains unchanged, a progressive reduction in dose requirement would be predicted if administered twice weekly by subcutaneous injection.

In conclusion, correction of anaemia with $\mathrm{r}-\mathrm{HuEpo}$ is associated with an objective improvement in exercise tolerance and a subjective improvement in physical fitness and health, including better school attendance. These benefits, together with the important cardiac benefits already reported, support the use of r-HuEpo in all children with end stage renal failure and anaemia.
We are grateful to the haematology and biochemistry departments of the Royal Victoria Infirmary and the electrocardiography department of Freeman Hospital, Newcastle upon Tyne for their help with this study. Also to Boehringer Mannheim (UK) who supplied human recombinant erythropoietin (Recormon) for the study and carried out the assays. KPM was funded by Newcastle District Research Committee and Child Kidney Fund.

1 Eschbach JW. Recombinant human erythropoietin in patients on hemodialysis: United States. In: Erslev AJ, Adamson JW, Eschbach JW, Winearls CG AJ, Arythropoietion, Eschbach JW, Winearls CG, eds. Erythropoietin; molecular, cellular, and clinical biology. Baltimore: John Hopkins University Press, 1991: 211-27. neous recombinant human erythropoietin in children undergoing continuous cycling peritoneal dialysis. f Pediatr 1989; 114: 550-4.

3 Montini G, Zacchello G, Baraldi E, et al. Benefits and risks of anemia correction with recombinant human erythropoietin in children maintained by hemodialysis. $\mathcal{f}$ Pediatr 1990; 117: 556-60.

4 Navarro M, Alonso A, Avilla JM, Espinosa L. Anemia of chronic renal failure: treatment with erythropoietin. Child Nephrol Urol 1991; 11: 146-51.

5 Morris KP, Skinner JR, Hunter S, Coulthard MG. Short term correction of anaemia with recombinant human erythropoietin and reduction of cardiac output in end stage renal failure. Arch Dis Child 1993; 68: 644-8.

6 Upton CJ, Tyrrell JC, Hiller EJ. Two minute walking distance in cystic fibrosis. Arch Dis Child 1988; 63: 1444-8.

7 Bruce RA, Kusumi F, Hosmer D. Maximal oxygen intake and nomographic assessment of functional aerobic impairment in cardiovascular disease. Am Heart $\mathcal{f} 1973$; 85: 546-2.

8 Bacon CJ, Cranage JD, Hierons AM, Rawlins MD, Webb JKG. Behavioural effects of phenobarbitone and phenytoin in small children. Arch Dis Child 1981; 56: 836-40.

9 Coulthard MG, Sharp J. PROD: peritoneal rapid overnight dialysis in children. Pediatr Nephrol 1989; 3: C218.

10 Morris KP, Watson S, Reid MM, Hamilton P, Coulthard MG. Assessing iron status in children with chronic renal failure on erythropoietin: which measurements should we use? Pediatr Nephrol (in press).

11 Armitage P, Berry G. The simple crossover design. Statistical methods in medical research. 2nd Ed. London: Blackwell Scientific Publications, 1987: 222-6.

12 Rigden SPA, Montini G, Morris M, et al. Recombinant human erythropoietin therapy in children maintained by haemodialysis. Pediatr Nephrol 1990; 4: 618-22.

13 Bommer J, Alexiou C, Muller-Buhl U, Eifert J, Ritz E. Recombinant human erythropoietin therapy in haemodialysis patients - dose determination and clinical experience. Nephrol Dial Transplant 1987; 2: 238-42.

14 Fabris F, Cordiano I, Randi ML, Casonato A, Montini G, Zacchello G. Effects of recombinant human erythropoietin on bleeding time, platelet number and function in children with end-stage renal disease maintained by haemodialysis. Pediatr Nephrol 1991; 5: 225-8.

15 Evans JHC, Brocklebank JT, Bowmer CJ, Ng PC. Pharmacokinetics of recombinant human erythropoietin in children with renal failure. Nephrol Dial Transplant 1991; 6: 709-14.

16 Macdougall IC, Roberts DE, Neubert P, Dharmasena AD, Coles GA, Williams JD. Pharmacokinetics of recombinant human erythropoietin in patients on continuous ambulatory peritoneal dialysis. Lancet 1989; i: 425-7.

17 Canadian Erythropoietin Study Group. Association between recombinant human erythropoietin and quality between recombinant human erythropoietin and quality
of life and exercise capacity of patients receiving of life and exercise capacity of pat

18 Warady BA, Sabath RJ, Smith CA, Alon U, Hellerstein S. Recombinant human erythropoietin therapy in pediatric patients receiving long-term peritoneal dialysis. Pediatr Nephrol 1991; 5: 718-23.

19 Campos A, Garin EH. Therapy of renal anemia in children and adolescents with recombinant human erythropoietin. Clin Pediatr (Phila) 1992; 31: 94-9.

20 Scigalla P [European Multicenter Study Group]. Effect of recombinant human erythropoietin treatment on renal anemia and body growth of children with end-stage renal anemia and body growth of children with

21 Macdougall IC, Davies ME, Hutton RD, et al. The treatment of renal anaemia of CAPD patients with recombinant human erythropoietin. Nephrol Dial Transplant 1990; 5: $950-5$.

22 Hutchison AJ, Ofsthun NJ, Howarth D, Gokal R. The effect of hemoglobin concentration on peritoneal mass transfer and drain volumes in continuous ambulatory peritoneal dialysis Peritoneal Dialysis International 1992; 12: 230-3.

23 Steinhauer HB, Lubrich-Birkner I, Dreyling KW, Hor WH, Schollmeyer P. Increased ultrafiltration after erythropoietin-induced correction of renal anemia in patients on continuous ambulatory peritoneal dialysis. Nephron 1989; 53: 91-2.

24 Korbet SM, Vonessa EF, Firanek CA. The effect of hematocrit on peritoneal transport. Am $\mathcal{f}$ Kidney Dis 1991; 18: 573-8.

25 Edmunds ME, Walls J, Tucker B et al. Seizures in haemodialysis patients treated with recombinant human erythropoietin. Nephrol Dial Transplant 1989; 4: 1065-9.

26 Teruel JL, Pascual J, Liano F, Gamez C, Fernandez M, Ortuno J. Long-term evolution of blood pressure in eryOrtuno J. Long-term evolution of blood pressure in ery-
thropoietin-treated patients on haemodialysis: evidence of 
two blood pressure responses (abstract). Nephrol Dial Transplant 1991; 6: 829.

27 Tomson CRV, Feehally J, Walls J. Crossover comparison of intravenous and subcutaneous erythropoietin in haemodialysis patients. Nephrol Dial Transplant 1992; 7 129-32.

28 Kampf D, Eckardt KU, Fischer HC, Schmalisch C, Ehmer B, Schostak M. Pharmacokinetics of recombinant human erythropoietin in dialysis patients after single and multiple subcutaneous administrations. Nephron 1992; 61: 393-8.

\section{Appendix}

Parental questionnaire together with the descriptions used at opposite ends of each linear analogue scale.

1. How long does he/she sleep each night (to nearest hour)?

2. How long does he/she sleep during the day (to nearest hour)?

3. How easy is it to get him/her settled at night? Very easy/very hard

4. How often does he/she wake up at night? Very often/never

5. How often does he/she cry? Very often/never

6. How is his/her general health at present? Very good/very bad

7. How active is helshe? Very active/not at all active

8. Does he/she appear anxious or worry a lot? Very often/never

9. How often does he/she have tantrums? Very often/never

10. How is his/her appetite? Very good/very poor
11. How fussy is he/she about his/her food? Very fussy/not at all fussy

12. How often does he/she vomit? Very often/never

13. How well does he/she get along with other children? Very well/not at all

14. Is he/she pleasant or bad tempered?

Very pleasant/very bad

15. During exercise does he/she get tired easily? Very easily/not at all easily

16. Is he/she generally happy at present? Very happy/very unhappy

17. How well does he/she do what he/she is told? Very well/not at all well

18. How breathless is he/she after exercise? Very breathless/not at all

19. How well does he/she concentrate on the job at hand? hand?

0. Does he/she

21. How is his/her school work at present? Very good/very poor

22. What is he/she like after school? Full of beans/very tired

23. How many days has he/she missed school through poor health in the last 3 months?

24. Have you noticed any overall change in him/her over the past 3 months?

Much better/much worse

25. Which do you think he/she has been receiving? r-HuEpo/placebo/not sure

(Questions 20-23 for schoolchildren only) 